\title{
Evaluating patterns of failure and salvage therapy for patients treated with primary stereotactic body radiation therapy for early stage non-small cell lung cancer
}

\author{
Megan Mezera ${ }^{1}$, Mahesh Chandrasekhar ${ }^{2}$, Goetz Kloecker ${ }^{3}$, Victor van Berkel ${ }^{4}$, Michael \\ Bousamra ${ }^{4}$, Neal E Dunlap ${ }^{1}$
}

1. Department of Radiation Oncology, University of Louisville, Louisville, KY, U.S.A. 2. School of Medicine, University of Louisville Louisville, KY, U.S.A. 3. Department of Internal Medicine, Division of Hematology Oncology, University of Louisville, Louisville, KY, U.S.A. 4. Department of Thoracic Surgery, University of Louisville School of Medicine, Louisville, KY, U.S.A.

Correspondence: Neal E Dunlap. Address: 529 South Jackson St. Department of Radiation Oncology, University of Louisville, Louisville, KY, U.S.A. 40241. Email: nedunl01@louisville.edu

Received: November 14, 2013 Accepted: February 13, 2014

Online Published: February 25, 2014

DOI : $10.5430 /$ jst.v4n2p4

URL: http://dx.doi.org/10.5430/jst.v4n2p4

\section{Abstract}

Objective: The purpose of this study is to evaluate patterns of failure after stereotactic body radiation therapy for early stage NSCLC and determine the role of salvage therapy on patient outcome.

Methods: Eighty-two consecutive medically inoperable patients treated with definitive SBRT for early-stage NSCLC were examined. Ninety-four percent of patients had biopsy proven disease, the remainder refused. The median biologic effective dose (BED) for the cohort was $100 \mathrm{~Gy}$ (range, 78-180 Gy). Recurrence was defined clinically by RECIST and FDG-PET or by pathologic diagnosis.

Results: The estimated median survival for the entire cohort was 29.2 months (range, $1-40.3$ ). The estimated 2 year overall survival (OS) was $65 \%$. The 2 year local recurrence free survival, locoregional recurrence free survival and distant metastatic free survival were $89 \%, 78 \%$ and $72 \%$, respectively. Of those with locoregional failure, ten were treated with salvage SBRT at a median time of 10 months from prior SBRT. Six patients were treated with systemic chemotherapy and 2 patients were offered palliative care. All patients treated with salvage SBRT remained controlled at a median time of 12 months. Treatment was well tolerated with 2 patients experiencing grade 2 pneumonitis, 1 patient experiencing grade 3 chest wall pain and 1 with rib fracture. OS was compared between 3 subgroups: no locoregional failure $(\mathrm{n}=65)$, locoregional failure without SBRT salvage $(\mathrm{n}=7)$ and locoregional failure with SBRT salvage $(\mathrm{n}=10)$. The estimated median survival 31.3 months, 14.3 months and 29.9 months, respectively $(p=0.007)$.

Conclusion: In our single institution patient cohort, SBRT in the salvage setting for locoregional recurrences after initial definitive SBRT for early-stage NSCLC is a potential option. Additional prospective data is needed.

\section{Key words}

Recurrence, Re-treatment, Inoperable, Lung cancer 


\section{I ntroduction}

Non-small cell lung cancer (NSCLC) is the most common cause of cancer death in the U.S. and worldwide, accounting for the highest cancer mortality rates for both men and women ${ }^{[1]}$. With the widespread use of computed tomography for diagnosis, an increasing proportion of NSCLC cases now present with localized early stage disease ${ }^{[2,3]}$. Surgical resection, either by lobectomy or pneumonectomy, has long been the standard of care for early stage NSCLC. However, many lung cancer patients are not surgical candidates due to common medical comorbidites including chronic obstructive pulmonary disease (COPD), coronary artery disease, and inadequate pulmonary reserve as determined by pulmonary function tests, resulting in unacceptably high levels of perioperative morbidity and mortality.

Stereotactic body radiation therapy (SBRT) is a non-invasive alternative to surgery for early-stage NSCLC patients who are medically inoperable or who refuse surgery. Multiple studies have demonstrated excellent tumor control and limited toxicity with the use of SBRT ${ }^{[3-5]}$. For patients with T1 and T2 tumors with a high operative risk, SBRT results in tumor control and overall survival comparable to reported results for surgery, with local control rates greater than $90 \%{ }^{[5-7]}$.

Despite the success of SBRT as a treatment modality for early-stage NSCLC, there is a subset of patients who develop intrathoracic recurrences after treatment without evidence of distant metastatic disease. An effective salvage therapy for this group of patients is typically limited. We examine our institutional experience of using SBRT for early stage NSCLC to define patterns of failure and outcomes after salvage therapy.

\section{Methods}

Between 2009 and 2012, 128 consecutive patients were treated with thoracic SBRT for NSCLC at the University of Louisville after institutional review board approval for an institutional protocol. Of those treated, 82 patients were identified as early stage and received definitive SBRT. The remaining patients were treated for oligometastatic lesions to the lung or mediastinum $(n=22)$ and recurrence after treatment for locally advanced NSCLC $(n=24)$. Primary tumors were defined as central $(n=15)$ or peripheral $(n=67)$ tumors by RTOG protocol 0236 as the primary tumor not touching a volume $2 \mathrm{~cm}$ in all directions around the proximal bronchial tree (distal $2 \mathrm{~cm}$ of the trachea, mainstem bronchi, and lobar bronchi). The median patient age was 73.1 years (range, 50.4-90.1 years). All patients underwent pre-SBRT computed tomography (CT) of the chest and abdomen for clinical staging. Positron emission tomography (PET) CT scan was obtained as part of the initial staging workup in all patients. Patients with abnormal fluorodeoxyglucose (FDG) uptake in the mediastinum, as characterized by a maximum standardized uptake value (SUV) $>2.5$, were not considered candidates for primary SBRT. Mediastinoscopy or endobronchial ultrasound was not performed on a routine basis unless the patient had suspicious findings on CT or PET. On the basis of imaging imaging, patients were classified as clinical stage according to American Joint Committee on Cancer staging (2010). Histologic confirmation of cancer was obtained in most patients by either tissue biopsy or cytology. Five patients either refused biopsy or were deemed high risk for biopsy.

Patients were treated with lung SBRT if they were considered to be medically inoperable by a multi-disciplinary team. Guidelines for inoperability were determined by the thoracic surgeon and typically included a predicted postoperative forced expiratory volume in 1 second $<30 \%$, severely reduced diffusion capacity $<40 \%$ predicted, a Karnofsky performance status of $<70 \%$, or severe cardiac disease according to the New York Heart Association functional classification. No patients received prior lung irradiation. All patients were required to have a Karnofsky Performance Status (KPS) $>70 \%$.

\subsection{Treatment planning and procedure}

All patients underwent a treatment planning scan using 4D CT imaging using the Varian RPM system. In patients who were unable to generate a usable pattern of respiration, biofeedback was incorporated with audiovisual devices. Patients were immobilized using a "frameless" semi-rigid evacuated bag system (Vac-Lok; MEDTEC, Orange City, IA). An 
isocenter was placed in the geometric center of the tumor. The internal tumor volume (ITV) was identified using each of the 10 phases generated by 4D CT imaging. The clinical target volume was identical to the ITV. The planning target volume (PTV) was created by expanding the ITV volume $0.5 \mathrm{~cm}$ in all directions. Normal tissue dose constraints, as recommended by the American Association of Physicists in Medicine (AAPM) Task Group (TG) 101, were followed ${ }^{[8]}$. The need for respiratory gating was determined by the treating physician but was typically employed for tumor motion $>1 \mathrm{~cm}$.

Treatment planning and target volume delineation was determined by both the thoracic surgeons and the radiation oncologists and performed using the Varian Eclipse treatment planning system (Varian Medical Systems, Palo Alto, CA). Patients were treated using volumetric modulated arc therapy (VMAT) with Rapid Arc. Heterogeneity corrections were used routinely. Successful treatment planning objects were based the criteria established in the RTOG 0813 and 0914 study protocols. Two partial arcs (0 - 270 degrees) were utilized on all patients for dose delivery. Daily cone beam CT (CBCT) was used for treatment alignment. Gating levels were verified by fluoroscopic imaging prior to treatment delivery. When respiratory gating was required, a gated VMAT technique was utilized.

The median prescribed dose in our cohort was $50 \mathrm{~Gy}$ (range, 42-60 Gy) in 3 to 5 fractions. The median biologic effective dose $(\mathrm{BED}$, alpha/beta $=10)$ for the cohort was $100 \mathrm{~Gy}$ (range, 78-180 Gy). The selection of total dose and fractionation to the primary tumor was determined by the treating physician or constraints to the adjacent normal tissues. The prescribed dose was not dictated by $\mathrm{T}$ stage or tumor size.

\subsection{Follow-up and evaluation}

After SBRT, follow-up was performed approximately 8 weeks after treatment and approximately every 3 months thereafter. CT of the chest was routinely obtained at 3-month intervals from the completion of radiotherapy. PET-CT was reserved to evaluate progressive changes on $\mathrm{CT}$ scans that were considered worrisome for local or regional failure. Local recurrence was defined as recurrence within the treated lobe of the lung. Tumor progression required both of the following conditions be met: a $20 \%$ increase in the smallest diameter of the lesion with at least a 5 -mm increase in diameter, as specified by RECIST 1.1 (9) and a $\geq 20 \%$ increase in the maximum SUV from the pretreatment value seen on PET-CT. Biopsy was performed as a confirmatory test for local or regional recurrence in select patients. Nodal recurrence and recurrences in the ipsilateral non-treated lobe was defined as regional recurrence. Metastatic recurrence or recurrence in the contralateral hemithorax was defined as distant systemic metastases. Toxicity was evaluated by the treating physician at each visit using the Common Terminology Criteria for Adverse Events v3.0 (CTCAEv3).

\subsection{Salvage therapy}

The decision for using salvage therapy was determined by the treating physician and multidisciplinary team. In patients treated with SBRT for salvage, the re-irradiation volume was defined as either in-field (occurring within the 50\% isodose line of the prior SBRT treatment) or out-of-field. Re-irradiation dose was determined by the physician. The median salvage dose used was 50Gy (range, 40Gy - 54Gy) with a median BED of 100Gy (range, 72Gy - 151Gy). Standard SBRT treatment planning procedure and delivery was utilized as described above. Patients treated with salvage chemotherapy was directed by the medical oncologist and was based on patient age, performance status and tumor histology.

\subsection{Statistical analysis}

The follow-up was determined from the date of the final SBRT treatment to calculate median follow-up and Kaplan-Meier outcome data, including local recurrence free survival (LRFS), nodal recurrence free survival (NRFS), locoregional recurrence free survival (LRRFS), distant metastasis free survival (DMFS) and overall survival (OS). SPSS 21 (Chicago, IL) was used for statistical analysis. A Cox regression analysis was performed to adjust outcomes according to patient-specific data using multiple variables analyzed simultaneously. 


\section{Results}

\subsection{Patient characteristics}

All patients received the prescribed dose. The median follow-up time for the entire cohort from the end of treatment was 24 months (range, 6.0-40.3 months). The median tumor size was $25 \mathrm{~mm}$ (range, $8-50 \mathrm{~mm}$ ). A majority of patients had Stage IA disease; T1a $(\mathrm{n}=35)$, T1b $(\mathrm{n}=24)$, T2a $(\mathrm{n}=19)$ and T3 due to parietal pleura involvement $(\mathrm{n}=4)$. Seventy-seven patients had an established tissue diagnosis; adenocarcinoma $(n=28)$, squamous cell carcinoma $(n=23)$, non-small cell carcinoma NOS $(n=22)$, adenosquamous carcinoma $(n=3)$ and large cell carcinoma $(n=1)$.

Pre-treatment pulmonary function test (PFT) were performed in approximately $50 \%$ of patients in our cohort. Of those with information available, the median forced expiratory volume over $1 \mathrm{sec}$ (FEV1) was 56\% predicted (range, 22\% $95 \%$ ) and median diffusion capacity of carbon dioxide (DLCO) was $29.5 \%$ predicted (range, $10 \%-66 \%$ ). The median value maximum pre-treatment SUV for the primary tumor was 4.0 (range, $1.3-17.5$ ).

\subsection{Treatment toxicity and response}

Of the 82 patients treated in our cohort, symptomatic pulmonary complications (grade $>1$ ) occurred in 10 patients $(12 \%)$, with 6 patients developing grade 2 pneumonitis, 2 patients with grade 3, 1 patient with grade 4 (necrotizing pneumonia) and 1 patient with grade 5 (pneumonia leading to death). Two patients experienced grade 2 esophagitis. Six patients experienced grade 1 dermatitis. Chest wall pain was fairly infrequent (13\%), with 5 patients experiencing grade 2 and 1 patient with grade 3.

The median time to maximal response based on RECIST 1.1 after SBRT was 6.8 months (range, 1.5-18.0 months). Response rate was assessed at 6 months after treatment. Eighteen patients (22\%) within the cohort had a radiographic complete response, whereas 50 patients $(61 \%)$ had a partial response. The remainder had stable disease.
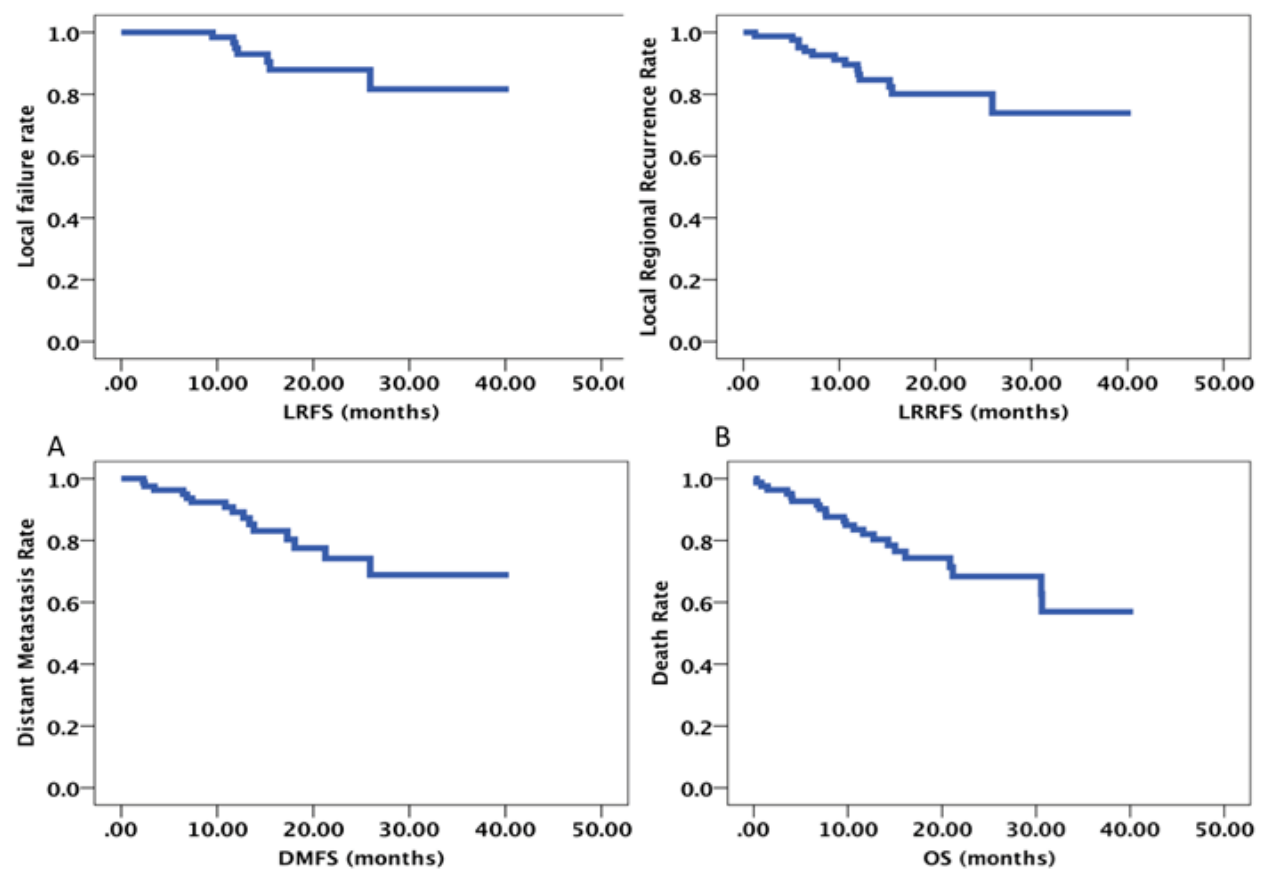

C

D

Figure 1. Local recurrence free survival (A), local regional recurrence free survival (B), distant metastasis free survival (C) and overall survival (D) for all patients treated with primary SBRT 
See Figure 1a-e for outcome data. The estimated median survival for the entire cohort was 29.2 months (range, 1 - 40.3). The estimated 1 and 2 year overall survival (OS) was $78 \%$ and $65 \%$, respectively. Seven patient (9\%) experienced local failure at a median time of 12.13 months (range, 9.50-14.2). Recurrence was documented by biopsy in 1 patient while imaging criteria was used to define the additional recurrences. The estimated 1 and 2 year local recurrence free survival (LRFS) was $94 \%$ and $89 \%$, respectively. Nine patients (11\%) experienced mediastinal or ipsilateral hilar lymph node failure at a median time of 5.8 months (range, 1.2 - 25.9). All nodal recurrences were documented by imaging criteria alone. The estimated 1 and 2 year nodal recurrence free survival (NRFS) was $90 \%$ and $87 \%$, respectively. The estimated 1 and 2 year locoregional recurrence free survival (LRRFS) was $84 \%$ and $78 \%$, respectively. Fifteen patients (18\%) experienced distant failure at a median time of 7.36 months (range, $2.3-25.9$ ). The estimated 1 and 2 year distant metastasis free survival (DMFS) was $89 \%$ and $72 \%$, respectively. In patients who experienced local recurrence, 2 developed synchronous nodal recurrence and 1 developed a synchronous distant recurrence.

Table 1a. Cox regression analysis for local recurrence

\begin{tabular}{|c|c|c|c|c|}
\hline & \multirow{2}{*}{$P$ value } & \multirow{2}{*}{ Hazard Ratio (HR) } & \multicolumn{2}{|c|}{$95.0 \%$ CI for HR } \\
\hline & & & Lower & Upper \\
\hline Age & .875 & 1.152 & .197 & 6.742 \\
\hline KPS & 695 & .000 & .000 & 3.557 \\
\hline Pack years & .369 & 1.358 & 696 & 2.649 \\
\hline Size & .854 & 1.351 & .054 & 33.585 \\
\hline Tstage & .971 & & & \\
\hline Tstage(1) & .875 & .890 & .876 & 20.768 \\
\hline Tstage(2) & .833 & .806 & .001 & 5.990 \\
\hline Tstage(3) & .898 & .780 & .076 & 9.046 \\
\hline SUV & .925 & .435 & .039 & 49.675 \\
\hline RTdose & .859 & 1.023 & .798 & 1.311 \\
\hline
\end{tabular}

Table 1b. Cox regression analysis for node recurrence

\begin{tabular}{lllcc} 
& P value & Hazard Ratio (HR) & \multicolumn{2}{c}{ 95.0\% CI for HR } \\
\cline { 3 - 4 } & & & Lower & Upper \\
\hline Age & .790 & 1.023 & .864 & 1.211 \\
KPS & .571 & .002 & .000 & 1.890 \\
Pack years & .740 & .994 & .956 & 1.032 \\
Size & .041 & 1.803 & 1.651 & 1.991 \\
Tstage & .098 & & & \\
Tstage(1) & .812 & .040 & .005 & 5.643 \\
Tstage(2) & .014 & .001 & .000 & .228 \\
Tstage(3) & .352 & .088 & .001 & 14.731 \\
SUV max & .894 & 1.036 & .618 & 1.735 \\
RTdose & .184 & .998 & .996 & 1.001 \\
\hline
\end{tabular}

Cox regression analysis was performed to identify factors that contributed to local recurrence, nodal recurrence, distant recurrence and overall survival (see Tables 1a-d). No factors were identified as significant predictors for local failure. 
Increasing tumor size was predictive for nodal recurrence (HR 1.803, 95\% CI $1.651-1.991)$. There were no predictors for DM. Decreasing KPS (HR 0.70, 95\% CI 0.001 - 0.946), increasing number of pack years (HR 1.965, 95\% CI 1.933 1.998), SUV $\max ($ HR $0.585,95 \%$ CI $0.393-.869$ ) and RT dose (HR $0.997,95 \%$ CI $0.994-0.999$ ) were predictive for OS.

Table 1c. Cox regression analysis for distant metastasis

\begin{tabular}{lllcc}
\hline & P value & Hazard Ratio (HR) & \multicolumn{2}{c}{$\mathbf{9 5 . 0 \%}$ CI for HR } \\
\cline { 4 - 5 } & & & Lower & Upper \\
\hline Age & .254 & .916 & .787 & 1.065 \\
KPS & .149 & 49.652 & .027 & 67.933 \\
Pack years & .349 & 1.014 & .985 & 1.043 \\
Size & .362 & .948 & .844 & 1.064 \\
Tstage & .493 & & & \\
Tstage(1) & .955 & 30.443 & .872 & 82.883 \\
Tstage(2) & .961 & 1.114 & .558 & 5.331 \\
Tstage(3) & .966 & 1.337 & .044 & 4.225 \\
SUV max & .083 & 1.094 & .988 & 1.210 \\
RTdose & .655 & .999 & .995 & 1.003 \\
\hline
\end{tabular}

Table 1d. Cox regression analysis for overall survival

\begin{tabular}{|c|c|c|c|c|}
\hline & \multirow{2}{*}{$P$ value } & \multirow{2}{*}{ Hazard Ratio (HR) } & \multicolumn{2}{|c|}{$95.0 \%$ CI for HR } \\
\hline & & & Lower & Upper \\
\hline Age & .024 & 1.145 & 1.018 & 1.288 \\
\hline KPS & .013 & .700 & .001 & .946 \\
\hline Pack Years & .037 & 1.965 & 1.933 & 1.998 \\
\hline Tumor Size & .590 & .971 & .870 & 1.082 \\
\hline Tstage & .406 & & & \\
\hline Tstage(1) & .794 & 1.708 & .031 & 94.614 \\
\hline Tstage(2) & .877 & .807 & .053 & 12.355 \\
\hline Tstage(3) & .132 & 7.661 & .542 & 108.342 \\
\hline SUV max & .008 & .585 & .393 & .869 \\
\hline RTdose & .010 & 997 & .994 & .999 \\
\hline
\end{tabular}

\subsection{Salvage treatment}

Eighteen patients ultimately experienced either a local or regional recurrence, of which ten patients were treated for salvage with SBRT. Of the remain 8 patients, 6 received palliative chemotherapy. The most common regimen was carboplatin/paclitaxel $(n=4)$ followed by carboplatin/pemetrexed $(n=2)$. Two patients were treated with palliative measures only. No patient treated with SBRT for salvage, received chemotherapy. Factors for determining which patient received salvage SBRT were examined including patient age, performance status, prior radiation dose, tumor location (in-field versus out-of-field), time from original SBRT and lack of distant metastasis. The only factor predictive for utilizing salvage SBRT as opposed to chemotherapy or palliative care was the absence of distant metastases $(p=0.05)$. 
The site of SBRT salvage was as follows: four patients were treated for in-field recurrence only, four patients were treated for out-of-field recurrence only and two patients were treated for both in-field and out-of-field recurrence. All out-of-field recurrences occurred in un-irradiated regional lymph nodes. Of those treated for nodal recurrence, 3 occurred in the ipsilateral mediastinum and 2 in the ipsilateral hilum. The median time from completion of the initial definitive SBRT to salvage SBRT treatment was 10.0 months (range, 2.6 - 16.6). Cumulative dose volume histogram (DVH) data was calculated to organs at risk. The median maximum chest wall dose was $60.33 \mathrm{~Gy}$ (range, $48.73-132.37$ ). The median volume receiving $30 \mathrm{~Gy}$ was $62 \mathrm{cc}$ (range, $15-120$ ). The median V20 for the cohort was $15 \%$ (range, $8-22$ ). The median dose to $5 \mathrm{cc}$ of the main bronchus was $32.45 \mathrm{~Gy}$ (range, $12.13-51.46$ ). The treatment was well tolerated with 2 patients experiencing grade 2 pneumonitis, 1 patient experiencing grade 3 chest wall pain and 1 with rib fracture. There was no correlation between DVH parameters and the development of treatment related toxicity.

At last follow up, the salvage SBRT site was controlled in all patients at a median time of 12 months from completion of salvage (range, 3.0 - 18.1). Using Kaplan-Meier analysis, OS was compared between 3 subgroups; no locoregional failure $(\mathrm{n}=65)$, locoregional failure without salvage SBRT $(\mathrm{n}=8)$ and locoregional failure with salvage SBRT $(\mathrm{n}=10)$. The estimated median survival 31.3 months, 14.3 months and 29.9 months, respectively. The estimated 2 year OS was $78 \%$, $50 \%$ and $80 \%$, respectively (Figure 2). Using the log rank test there was statistically significant lower survival in patients with locoregional failure without salvage SBRT when compared to those with salvage SBRT $(p=0.007)$. There was no difference in survival between those with no locoregional failure and those with locoregional failure with salvage SBRT ( $p$ $=0.783$ ). When controlled for distant metastatic disease the difference remained significant.

Figure 2. Survival data comparing those with no local regional failure, local regional failure without salvage and local regional failure with salvage

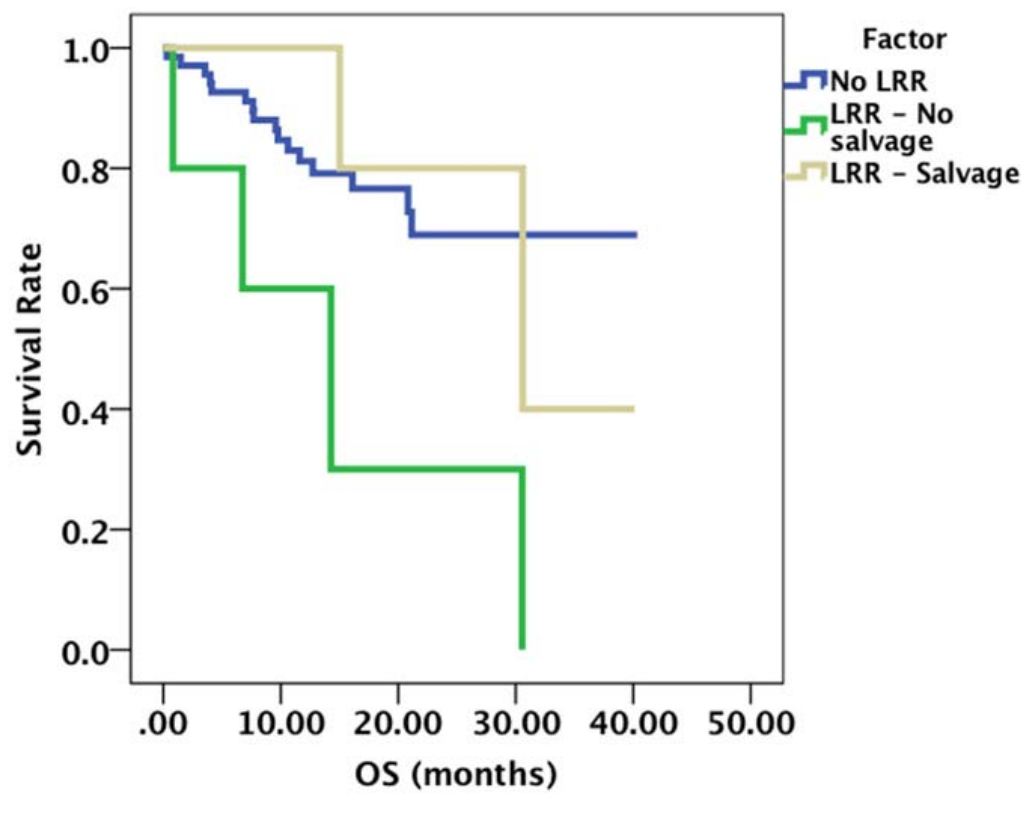

\section{Discussion}

Multiple studies have confirmed the safety and efficacy of using SBRT for definitive treatment of medically inoperable early-stage NSCLC ${ }^{[3,5,7]}$. Local control rates have been reported between $85 \%-97 \%$, with local-regional control rates of approximately $87 \%$ at 3 years ${ }^{[5]}$. Biopsy by means of a transthoracic or transbronchial approach is often contraindicated and therefore defining pathologic failure is somewhat complicated. Defining failures in a non-surgical population is based typically on clinical assessment. In our patient cohort, only 1 patient was able to undergo biopsy for recurrence confirmation while the remainder were diagnosed clinically. The ability to distinguish between tumor recurrence and 
mass-like consolidation after lung SBRT is a significant clinical problem. Applying standard RECIST 1.1 criteria to mass-like consolidation is very misleading and can lead to an overestimation of tumor recurrence. Data from a recent publication indicate that current RECIST criteria may have positive predictive value as low as $28 \%$, leading to a high number of false positive results ${ }^{[10]}$. Incorporating additional anatomic and metabolic imaging data, although not $100 \%$ specific, may help to improve the diagnostic accuracy of recurrences ${ }^{[10-13]}$.

Salvage options after lung SBRT are somewhat limited and chemotherapy is often recommended. Aggressive local therapy can be considered in a select group of patients. Recent publications outline the feasibility of surgical resection in highly selected patients ${ }^{[14,15]}$. Chen et al. ${ }^{[14]}$ reviewed 144 initially operable patients treated with SBRT. Local recurrence was identified in 24 patients, of which 5 patients underwent surgical salvage with a lobectomy and lymph node dissection range from $8-89$ months after initial SBRT. At a median follow up of 27 months, all patients were alive with minimal operative morbidity. Similarly, Neri et al. ${ }^{[15]}$ reviewed 146 operable patients with both stage I NSCLC and oligometastatic disease treated with SBRT. Eighteen patients had local tumor recurrence, of which 7 underwent surgical salvage (lobectomy or segmentectomy). Five patients had a complete resection with minimal surgical morbidity.

Despite the potential for surgical salvage, most patients receiving SBRT to the malignant lesions in the lung are not eligible for surgery because of baseline pulmonary function, underlying cardiac disease, marginal performance status, or a combination of these factors. These factors also contribute to limited options for salvage in the subset who fail after SBRT. In patients who are not eligible for surgery, salvage has been limited to chemotherapy, further standard radiation therapy, or observation. SBRT has been integrated into salvage algorithms in patients with recurrent, locally advanced lung cancer at some centers ${ }^{[16]}$. MD Anderson Cancer Center reviewed 246 patients treated with SBRT between $2004-2008$. Thirty-six patients had received prior thoracic radiotherapy. The median dose delivered at the time of initial treatment was 61.5 Gy (range, 30-79.2 Gy), with a majority receiving chemotherapy. At a median time from prior RT of 22 months, $50 \mathrm{~Gy}$ was delivered in 4 fractions for 11 in-field and 25 out of field relapses. The 2 year overall survival, progression free survival and local control were 59\%, 26\%, and 92\%, respectively. Treatment toxicity was acceptable, with $33 \%$ experiencing grade 3 toxicity and no patients developing grade 4 or 5 toxicity.

Despite the apparent favorable results in the retreatment setting for locally advanced disease, SBRT salvage is yet to be widely accepted as a means of salvage after initial definitive SBRT. Our data suggests that locoregional recurrence after SBRT in a highly selected group of patients can be salvaged safely using an additional course of SBRT. Although the follow up after salvage SBRT is relatively short (median 12 months) in this single institution, retrospective series, effective salvage was achieved for both in-field parenchymal lung recurrences and out-of-field nodal recurrences, with no evidence of progression to date. Additionally, overall survival was improved in patients undergoing salvage SBRT to levels equivalent to patients without locoregional failure (29.9 months vs. 31.3 months, $p=0.783$ ) and superior to chemotherapy or observation (29.9 months vs 14.3 months, $p=0.007)$. When analyzing patient selection (initial T stage, KPS, comorbidities, smoking status, distant metastasis, etc) for salvage SBRT, absence of distant metastasis was the only significant factor. When the presence of distant metastasis was controlled for in the survival analysis, no significant changes occurred. This may suggest that the improvement in overall survival is likely attributable to SBRT salvage and thus may have a role in patients with locoregional disease.

The toxicity profile of salvage SBRT appears to be favorable in the context of high local regional control and improved survival. Although early toxicity is relatively low, additional follow up is needed to ascertain late toxicity in this initial cohort. Chest wall toxicity was the most significant toxicity following salvage SBRT, with one patient developing a rib fracture. The mechanism of chest wall injury from SBRT is complex and multifactorial, but several studies have shown that increasing radiation dose correlates with increased incidence of severe chest wall pain and rib fracture ${ }^{[17-19]}$. In the retreatment setting, the incidence of radiation-induced chest wall pain is likely higher and precautions should be taken to limit chest wall dose to as low as reasonably possible without compromising tumor coverage. 


\section{Conclusions}

In our cohort, local, regional and distant recurrence is similar to previously published reports. Early results from our institution demonstrate that SBRT in the salvage setting for locoregional recurrences after initial definitive SBRT appears favorable with minimal toxicity for appropriately selected patients when used cautiously with close follow up. Our data is limited by the retrospective nature of the study and limited follow up. Long term results and prospective studies are needed to assess late toxicity and control rates.

\section{Conflicts of I nterest}

Megan Mezera, MD: No conflicts of interest or disclosures.

Mahesh Chandrasekhar, BS: No conflicts of interest or disclosures.

Geotz Kloecker: Dr. Kloecker currently receives payment for clinical lectures from Eli Lilly, Amgen and Genentech. Victor van Berkel, MD: No conflicts of interest or disclosures.

Michael Bousamra, MD: Dr. Bousamra has received money paid to self for legal consulting work within the last year. He has also received payment for telemedicine consultation approximately 5 years ago.

Neal Dunlap, MD: Dr. Dunlap has received money from the Institute for Medical Education for CME related activities within the last year.

\section{Author contributution}

Megan Mezera: Dr. Mezera performed data acquisition and analysis.

Mahesh Chandrasekhar: Performed data acquisition and analysis. He also worked on manuscript preparation.

Geotz Kloecker: Performed data analysis and manuscript preparation.

Victor Van Berkel: Involved with manuscript preparation and data analysis.

Michael Bousamra: Involved with manuscript preparation and data analysis.

Neal Dunlap: Corresponding author involved with data analysis and manuscript preparation.

\section{References}

[1] Siegel R, Naishadham D, Jemal A. Cancer statistics. CA Cancer J Clin. 2013; 63(1): 11-30. http://dx.doi.org/10.3322/caac.21166

[2] Shields, TW. Surgical therapy for carcinoma of the lung. Clin Chest Med.1993; 14: 121-147.

[3] Fakiris AJ, McGarry RC, Yiannoutsos CT, Papiez L, Williams M, Henderson MA, et al. Stereotactic body radiation therapy for early-stage non-small-cell lung carcinoma: four-year results of a prospective phase II study. Int J Radiat Oncol Biol Phys. 2009; 75(3): 677-682. http://dx.doi.org/10.1016/j.ijrobp.2008.11.042

[4] Bradley J, Graham MV, Winter K, Purdy JA, Komaki R, Roa WH, et al. Toxicity and outcome results of RTOG 9311: a phase I-II dose-escalation study using three-dimensional conformal radiotherapy in patients with inoperable non-small-cell lung carcinoma. Int J Radiat Oncol Biol Phys. 2005; 61(2): 318-328. http://dx.doi.org/10.1016/j.ijrobp.2004.06.260

[5] Timmerman RD, Paulus R, Galvin J, Michalski J, Straube W, Bradley J, et al. Stereotactic body radiation therapy for inoperable early stage lung cancer. JAMA. 2010; 303(11): 1070-1076. http://dx.doi.org/10.1001/jama.2010.261

[6] Baumann P, Nyman J, Hoyer M, Wennberg B, Gagliardi G, Lax I, et al. Outcome in a prospective phase II trial of medically inoperable stage I non-small-cell lung cancer patients treated with stereotactic body radiotherapy. J Clin Oncol. 2009; 27 : 3290-3296. http://dx.doi.org/10.1200/JCO.2008.21.5681

[7] Onishi H, Shirato H, Nagata Y, Hiraoka M, Fujino M, Gomi K, et al. Hypofractionated stereotactic radiotherapy (HypoFXSRT) for stage I non-small cell lung cancer: Updated results of 257 patients in a Japanese multi-institutional study. J Thorac Oncol. 2007; 2:S94-S100. http://dx.doi.org/10.1097/JTO.0b013e318074de34

[8] Benedict, SH, Yenice KM, Followill D, Galvin JM, Hinson W, Kavanagh B, et al. "Stereotactic body radiation therapy: the report of AAPM Task Group 101." Medical physics. 2010; 37: 4078. http://dx.doi.org/10.1118/1.3438081

[9] Eisenhauer EA, Therasse P, Bogaerts J, Schwartz LH, Sargent D, Ford R, et al. New response evaluation criteria in solid tumours: revised RECIST guideline (version 1.1) Eur J Cancer. 2009; 45: 228-245. http://dx.doi.org/10.1016/j.ejca.2008.10.026 
[10] Dunlap NE, Yang W, McIntosh A, Sheng K, Benedict SH, Read PW and Larner JM, et al. Computed tomography-based anatomic assessment overestimates local tumor recurrence in patients with mass-like consolidation after stereotactic body radiotherapy for early-stage non-small cell lung cancer. Int J Radiat Oncol Biol Phys. 2012 Dec 1;84(5):1071-7. http://dx.doi.org/10.1016/j.ijrobp.2012.01.088

[11] Takeda T, Takeda A, Kunieda E, Ishizaka A, Takemasa K, Shimada K, et al. Radiation injury after hypofractionated stereotactic radiotherapy for peripheral small lung tumors: serial changes on CT. AJR Am J Roentgenol. 2004; 182: 1123-1128. http://dx.doi.org/10.2214/ajr.182.5.1821123

[12] Patz EF Jr., Lowe VJ, Hoffman JM, Paine SS, Harris LK and Goodman P C. Persistent or recurrent bronchogenic carcinoma: detection with PET and 2-[F-18]-2-deoxy-D-glucose. Radiology. 1994; 191: 379-382.

[13] Hoopes DJ, Fletcher J, Tann M, Fletcher JW, Forquer JA, Lin PF, Lo SS, et al. A prospective trial of serial FDG-PET in patients with medically inoperable stage I non-small lung cancer treated with hypofractionated stereotactic body radiotherapy (SBRT) (Abstr.). Int J Radiat Oncol Biol Phys. 2006; 66(suppl): S25-S26. http://dx.doi.org/10.1016/j.ijrobp.2006.07.1344

[14] Chen F, Matsuo Y, Yoshizawa A, Sato T, Sakai H, Bando T, et al Salvage lung resection for non-small cell lung cancer after stereotactic body radiotherapy in initially operable patients. J Thorac Oncol. 2010; 5: 1999-2002. http://dx.doi.org/10.1097/JTO.0b013e3181f260f9

[15] Neri S, Takahashi Y, Terashi T, Hamakawa H, Tomii K, Katakami N, et al. Surgical treatment of local recurrence after stereotactic body radiotherapy for primary and metastatic lung cancers. J Thorac Oncol. 2010; 5: 2003-2007. http://dx.doi.org/10.1097/JTO.0b013e3181f8a785

[16] Kelly P, Balter PA, Rebueno N, Sharp HJ, Liao Z, Komaki R, et al. Stereotactic body radiation therapy for patients with lung cancer previously treated with thoracic radiation. Int J Radiat Oncol Biol Phys. 2010: 1387-1393. http://dx.doi.org/10.1016/j.ijrobp.2009.09.070

[17] Dunlap NE, Cai J, Biedermann GB, Yang W, Benedict SH, Sheng K, et al. Chest wall volume receiving > 30 Gy predicts risk of severe pain and/or rib fracture after lung stereotactic body radiotherapy. Int J Radiat Oncol Biol Phys. 2010 Mar 1; 76(3): 796-801. http://dx.doi.org/10.1016/j.ijrobp.2009.02.027

[18] Mutter RW, Liu F, Abreu A, Yorke E, Jackson A and Rosenzweig KE. Dose-volume parameters predict for the development of chest wall pain after stereotactic body radiation for lung cancer. Int J Radiat Oncol Biol Phys. 2012 Apr 1; 82(5): $1783-90$. http://dx.doi.org/10.1016/j.ijrobp.2011.03.053

[19] Lloyd S, Decker RH, Evans SB. Bone scan findings of chest wall pain syndrome after stereotactic body radiation therapy: implications for the pathophysiology of the syndrome. J Thorac Dis. 2013 Apr; 5(2): E41-4. 